CLINICAL STUDY

\title{
Family history of diabetes and distribution of class II HLA genotypes in children with newly diagnosed type 1 diabetes: effect on diabetic ketoacidosis
}

\author{
Anne Hekkala, Jorma Ilonen ${ }^{1,2}$, Mikael Knip ${ }^{3,4,5}$, Riitta Veijola and the Finnish Paediatric Diabetes Register* \\ Department of Paediatrics, Institute of Clinical Medicine, University of Oulu, PO Box 5000, 90014 Oulu, Finland, ${ }^{1}$ Immunogenetics Laboratory, \\ University of Turku, Turku, Finland, ${ }^{2}$ Department of Clinical Microbiology, University of Eastern Finland, Kuopio, Finland, ${ }^{3}$ Children's Hospital, \\ University of Helsinki and Helsinki University Central Hospital, Helsinki, Finland, ${ }^{4}$ Department of Paediatrics, Tampere University Hospital, Tampere, \\ Finland and ${ }^{5}$ Folkhälsan Research Center, Helsinki, Finland
}

(Correspondence should be addressed to A Hekkala; Email: anne.hekkala@oulu.fi)

*(The members of Finnish Diabetes Register are listed in the supplementary data available online)

\begin{abstract}
Objective: Our purpose was to assess whether family history of diabetes or the HLA-DR-DQ genotype of the index case was associated with the frequency of diabetic ketoacidosis (DKA) at diagnosis of childhood type 1 diabetes.

Patients and methods: The study cohort comprised 1518 children aged $<15$ years and diagnosed with type 1 diabetes in Finland in 2002-2005. Family history of type 1 and type 2 diabetes among firstdegree relatives (FDRs) and grandparents was assessed at diagnosis. HLA-DR-DQ genotypes were analysed using time-resolved fluorometry.

Results: In total, 12.6 and $1.7 \%$ of children had at least one FDR affected with type 1 or type 2 diabetes, respectively, and 6.6 and $34.8 \%$ had at least one grandparent with type 1 or type 2 diabetes. DKA (pH $<7.30$ ) occurred less frequently in children having a type 1 diabetes affected FDR (7.4 vs 20.5\%, $P<0.001)$. Type 2 diabetes among the parents or grandparents had no such effect. Lower risk HLA genotypes were observed to predispose to DKA $(P<0.024)$. In a logistic regression analysis, the risk of DKA was independently associated with the absence of a family member affected by type 1 diabetes, the presence of a low-risk HLA genotype and older age at diagnosis (odds ratio 3.23, 1.45 and 1.07 respectively).

Conclusion: The presence of type 1 diabetes in an FDR is associated with an decreased risk of DKA at diagnosis. The rate of DKA seems to be higher in children with lower HLA-conferred risk for type 1 diabetes.
\end{abstract}

European Journal of Endocrinology 165 813-817

\section{Introduction}

The frequency of diabetic ketoacidosis (DKA) at diagnosis of type 1 diabetes is lower in countries with a high incidence of the disease (1). A decreasing rate of DKA at diagnosis has been observed over time (2-4), and it has been postulated that increasing medical information and awareness might have resulted in changes in the clinical presentation of type 1 diabetes in developed countries $(1,5)$.

Familial aggregation of type 1 diabetes is a wellestablished phenomenon, and lower frequency of DKA at diagnosis in children having an affected first-degree relative (FDR) has been reported $(4,6)$. Specific HLA class II gene alleles at the HLA-DRB1, DQA1 and DQB1 loci are strongly associated with type 1 diabetes (7) and are more frequent in patients presenting with the disease in childhood than in adulthood $(6,8,9)$. However, little is known about the association between various HLA class II genotypes and the risk of DKA at disease onset, although this might be clinically important (10). The high-risk HLA-DQB ${ }^{*} 02 /{ }^{*} 0302$ genotype has been reported to be more frequent in children diagnosed $<2$ years, who also more often present with DKA at diagnosis (11). In Japan and China, specific HLA-DR-DQ genotypes have been associated with autoantibody-negative diabetes, fulminant clinical symptoms and severe DKA $(12,13)$.

The incidence of childhood type 1 diabetes is the highest in the world in Finland reaching 64.2/100 000 person-years in 2005 (14). The Finnish Paediatric Diabetes Register and Biobank was established in 2002 to collect clinical and biochemical data on children with newly diagnosed disease and their families. The purpose 
of this study was to investigate the effect of the family history of diabetes on the frequency of DKA at diagnosis and explore possible associations between HLA genotypes and the rate of DKA.

\section{Materials and methods}

The study cohort comprised children aged $<15$ years who had been diagnosed with type 1 diabetes in Finland between June 1, 2002 and May 31, 2005. In our country, all children developing type 1 diabetes under the age of 15 years are treated in paediatric units and such children and their families are invited to take part in the Finnish Paediatric Diabetes Register and the associated Biobank (see Supplementary data, see section on Supplementary data given at the end of this article for full details of the participating doctors and nurses). The doctors and nurses treating newly diagnosed patients have supplied the clinical and the laboratory data for the register. The register protocol has been approved by the Ethics Committee of the Hospital District of Helsinki and Uusimaa. The current analysis was approved by the local ethics committee and the Steering Committee of the register.

Information on the family history of type 1 and type 2 diabetes was obtained by interviewing the parents. FDR included the mother, the father and the full siblings of the affected child. In addition, the history of diabetes in the grandparents was registered. Gestational diabetes, monogenic diabetes or unclassified diabetes was not included in the current analysis. Definition of type 1 diabetes was based on diagnosis at young age and/or persistent requirement of exogenous insulin from the diagnosis. DKA was defined as blood $\mathrm{pH}<7.30$ and severe DKA as $\mathrm{pH}<7.10$ (15).

$H L A-D R-D Q$ genotypes were analysed centrally as described earlier (7). According to various HLA DRB1$D Q A 1-D Q B 1$ haplotype combinations, genotype conferring strongly increased, moderately increased, slightly increased, neutral and decreased risk for type 1 diabetes, were determined (Table 1). DKA frequencies were compared between the five HLA-associated risk groups and also between two larger categories, the first including subjects with strong- or moderate-risk genotypes and the other subjects with genotypes conferring slightly increased, neutral or decreased disease risk.

Data analyses were performed with SPSS for Windows Statistical Software (version 12.0; SPSS, Inc., Chicago, IL, USA). Student's two-tailed $t$-test for independent samples was used when comparing variables with normal distribution between the two groups. Mann-Whitney $U$ test was applied for unequally distributed variables. Distributions were analysed by cross-tabulation and $\chi^{2}$ statistics. To assess whether the frequency of DKA was related to age, sex, HLA genotype and type 1 or type 2 diabetes in FDR, the logistic regression model was used, and the results are
Table 1 The frequencies (\%) of HLA risk categories and frequencies of diabetic ketoacidosis (DKA) and severe DKA in different risk categories. Linear-by-linear association analysis was used to compare DKA frequencies between various risk groups. Alleles in parenthesis have not been analysed but can be deduced based on allelic associations in these haplotypes.

\begin{tabular}{lcc}
\hline $\begin{array}{l}\text { HLA-associated risk groups, } \\
n=1064 \text { and frequencies \% }\end{array}$ & DKA (\%) & $\begin{array}{c}\text { Severe } \\
\text { DKA (\%) }\end{array}$ \\
\hline Strongly increased risk ${ }^{\mathrm{a}}, 23.2 \%$ & 16.5 & 1.7 \\
Moderately increased risk $^{\mathrm{b}}, 45.2 \%$ & 17.9 & 3.6 \\
Slightly increased risk $^{\mathrm{c}}, 12.8 \%$ & 21.6 & 6.0 \\
Neutral risk $^{\mathrm{d}}, 15.2 \%$ & 23.3 & 6.3 \\
Decreased risk $^{\mathrm{e}}, 3.6 \%$ & 27.0 & 2.7 \\
$P$ value & 0.024 & 0.031 \\
\hline
\end{tabular}

aHeterozygosity for the two risk-associated haplotypes $D R B 1^{*} 0401 / 2 / 4 / 5 / 8$ $D Q A 1^{*} 03-D Q B 1^{*} 0302 / 4$ and [DRB1*03]-DQA ${ }^{*} 05-D Q B 1^{*} 02$.

${ }^{b}$ Above risk haplotypes as homozygous or $D R B 1^{*} 0401 / 2 / 4 / 5 / 8-D Q A 1^{*} 03-$ $D Q B 1^{*} 0302 / 4$ combined with a neutral haplotype, or the [DRB1*03]$D Q A 1^{*} 05-D Q B 1^{*} 02 /\left[D R B 1^{*} 09\right]-D Q A 1^{*} 03-D Q B 1^{*} 03$ genotype.

${ }^{\circ}\left[D R B 1^{*} 03\right]-D Q A 1^{*} 05-D Q B 1^{*} 02$ with a neutral haplotype or the $D R B 1^{*} 0401 / 2 / 5 / 8-D Q A 1^{\star} 03-D Q B 1{ }^{*} 0302 / 4 /\left[D R B 1{ }^{*} 1301\right]-\left[D Q A 1{ }^{*} 01\right]-$ $D Q B 11^{*} 0603$ genotype.

${ }^{d}$ Genotypes where a risk haplotype is combined with a protective one (DRB1*15-[DQA1*01]-DQB1*0602, [DRB1*11/12/13]-DQA1*05-DQB1* 0301, [DRB1*14]-[DQA1*01]-DQB1*0503, DRB1*07-DQA1*0201-DQB1* 0303, DRB1*0403-[DQA1*03]-DQB1*0302/4 and [DRB1*1301]-[DQA1*01]$D Q B 1^{*} 0603$ (except the combination in c)) or combinations of two neutral haplotypes.

${ }^{e}$ Combinations of two protective haplotypes or a protective haplotype associated with a neutral one.

reported as odds ratio (OR) and $95 \%$ confidence interval (CI). A two-tailed $P$ value of $<0.05$ was considered to indicate statistical significance.

\section{Results}

The study cohort included 1518 children diagnosed with type 1 diabetes below the age of 15 years (851 boys; $56.1 \%$ ) and participating in the Finnish Paediatric Diabetes register. These children represented $91.7 \%$ of all children diagnosed with type 1 diabetes during the 3-year period 2002-2005 in Finland (16). The mean age at diagnosis was 7.9 years (range $0.28-14.99$ ). At the time of diagnosis 94 children $(6.2 \%)$ were $<2$ years and a total of 398 children $(26.4 \%)$ were $<5$ years. Five hundred and eighty-three children $(38.6 \%)$ were between 5.0 and 9.99 years of age and 527 children $(35.0 \%)$ were between 10.0 and 14.99 years. The overall frequency of DKA in the study cohort was $19.2 \%$ (in 280 out of 1455 children) and the frequency of severe DKA was $4.1 \%$ (in 59 out of 1455 children). In this study, one child an 8-year-old girl, died of cerebral oedema at diagnosis.

The diabetes status of the FDRs were available for a total of 1384 children $(91.2 \%)$, and $12.6 \%(n=174)$ had at least one FDR affected by type 1 diabetes (Table 2). In $154(88.5 \%)$ of these families there was only one FDR with type 1 diabetes, whereas in 20 families $(11.5 \%)$ there were at least two such family 
Table 2 Proportion of newly diagnosed children ( $<15$ years) with a family history of diabetes.

\begin{tabular}{lccc}
\hline \multirow{2}{*}{$\begin{array}{l}\text { Family member } \\
\text { with diabetes }\end{array}$} & \multicolumn{3}{c}{ Type of diabetes in the given family member(s) } \\
\cline { 2 - 4 } & Type 1 diabetes & Type 2 diabetes & Type 1 or type 2 diabetes \\
\hline Father & $5.8 \%(81 / 1396)$ & $1.1 \%(16 / 1396)$ & $6.9 \%(97 / 1396)$ \\
Mother & $2.8 \%(40 / 1414)$ & $0.6 \%(8 / 1414)$ & $3.4 \%(48 / 1414)$ \\
Sibling & $4.9 \%(69 / 1418)$ & & $4.9 \%(69 / 1418)$ \\
Multiple siblings & $0.4 \%(6 / 1418)$ & & $0.4 \%(6 / 1418)$ \\
FDR & $12.6 \%(174 / 1384)$ & $1.7 \%(24 / 1384)$ & $14.1 \%\left(195^{\mathrm{a}} / 1386\right)$ \\
Paternal grandfather & $1.5 \%(19 / 1249)$ & $10.7 \%(134 / 1249)$ & $12.2 \%(153 / 1249)$ \\
Paternal grandmother & $1.0 \%(13 / 1285)$ & $8.8 \%(113 / 1285)$ & $9.8 \%(126 / 1285)$ \\
Maternal grandfather & $2.4 \%(31 / 1296)$ & $11.8 \%(153 / 1296)$ & $14.2 \%(184 / 1296)$ \\
Maternal grandmother & $1.0 \%(14 / 1331)$ & $8.2 \%(109 / 1331)$ & $9.2 \%(123 / 1331)$ \\
Any grandparent & $6.6 \%(76 / 1159)$ & $34.8 \%(429 / 1232)$ & $39.3 \%\left(488^{\mathrm{a}} / 1242\right)$ \\
FDR and/or grandparents & $19.7 \%\left(233^{\mathrm{b}} / 1182\right)$ & $36.0 \%\left(442^{\mathrm{C}} / 1229\right)$ & $48.3 \%\left(609^{\mathrm{a}} / 1262\right)$ \\
\hline
\end{tabular}

anformation from families in which at least one subject was known to be diagnosed with type 1 or type 2 diabetes.

${ }^{\mathrm{b}}$ There were $17(1.2 \%)$ children having both a FDR and a grandparent with type 1 diabetes.

'There were 11 children having both a FDR and a grandparent with type 2 diabetes.

members. Detailed data on family history of diabetes is presented in Table 2 .

Children $<2$ years of age had more often a father affected with type 1 diabetes than older children (11.2 vs $5.4 \%, P=0.024)$. In total, children $<2$ years had more frequently FDRs with type 1 diabetes (19.1 vs $12.1 \%, P=0.055)$ and FDR or grandparents affected with type 1 diabetes ( 31.1 vs $19.0 \%, P=0.011)$. When comparing age groups $0-4.99,5.0-9.99$ and 10.0-14.99 years, no differences were observed in the proportion of subjects with affected relatives $(12.8,13.4$ and $11.4 \%$ respectively).

DKA occurred less frequently among children having FDRs affected with type 1 diabetes (7.4 vs $20.5 \%$, $P<0.001$; Table 3). Furthermore, children with affected FDRs tended to have less frequently severe DKA at diagnosis than other children ( 1.2 vs $3.9 \%, P=0.082)$. None of the 65 children having a sibling with type 1 diabetes had severe DKA at diagnosis. In subjects $<2$ years and an affected FDR the frequency of DKA was $18.8 \%$ compared with $30.6 \%$ in those without an affected FDR $(P=0.344)$. The frequency of DKA tended to be lower in children with a grandparent with type 1 diabetes (Table 3). A lower frequency of DKA was observed in children with a parent with type 2 diabetes (0 vs $19.4 \%$ ), although the result was not statistically significant $(P=0.194)$. Diagnosis of type 2 diabetes in the grandparents had no effect on the frequency of DKA at diagnosis of type 1 diabetes in their grandchildren (Table 3).

HLA-associated genetic risk was defined in 1064 children (70.1\%; Table 1). Boys had less often HLA genotypes conferring strong or moderate disease risk (64.9 vs $73.2 \%, P=0.004$ ). Also, the proportion of children carrying strong- or moderate-risk HLA genotype tended to be lower in the oldest age group $(70.8 \%$

Table 3 Frequency of DKA defined as $\mathrm{pH}<7.30$ in children at diagnosis of type 1 diabetes. Data given separately for subjects with only a family member with type 1 diabetes (type 1 diabetes + and type 2 diabetes - ), only type 2 diabetes (type 1 diabetes - and type 2 diabetes +) or no diabetes (type 1 diabetes - and type 2 diabetes -). The data concerning the cases having any FDR, any grandparent or either one affected with diabetes has been marked bold.

\begin{tabular}{|c|c|c|c|c|c|}
\hline \multirow[b]{2}{*}{$\begin{array}{l}\text { Family member } \\
\text { with diabetes }\end{array}$} & \multicolumn{5}{|c|}{$\begin{array}{c}\text { Frequency of DKA }(\%(95 \% \mathrm{Cl})) \text { among the index cases by the presence and type of diabetes in the } \\
\text { given relatives }\end{array}$} \\
\hline & $\begin{array}{l}\text { Type } 1 \text { diabetes }+ \text { and } \\
\text { type } 2 \text { diabetes }-\end{array}$ & $\begin{array}{l}\text { Type } 1 \text { diabetes }- \text { and } \\
\text { type } 2 \text { diabetes }+\end{array}$ & $\begin{array}{l}\text { Type } 1 \text { diabetes }- \text { and } \\
\text { type } 2 \text { diabetes - }\end{array}$ & $P$ value $^{\mathrm{a}}$ & $P$ value ${ }^{\mathrm{b}}$ \\
\hline Father & $7.6(1.8-13.4)$ & $12.5(0-39.0)$ & $19.7(17.5-21.2)$ & 0.008 & 0.473 \\
\hline Mother & $5.0(0-11.8)$ & 0 & $19.4(7.3-21.5)$ & 0.022 & 0.194 \\
\hline Sibling & $7.7(1.2-14.2)$ & - & $19.5(17.4-21.6)$ & 0.018 & - \\
\hline Any FDR & $7.4(3.4-11.4)$ & $11.1(0-25.6)$ & $20.5(18.2-22.8)$ & $<0.001$ & 0.328 \\
\hline Paternal grandfather & $16.7(0-33.4)$ & $18.9(10.8-27.0)$ & $19.6(17.3-22.1)$ & 0.754 & 0.861 \\
\hline Paternal grandmother & 0 & $19.1(15.6-26.4)$ & $19.6(17.3-21.9)$ & 0.075 & 0.892 \\
\hline Maternal grandfather & $7.4(0-17.3)$ & $19.5(13.5-25.8)$ & $19.1(16.8-21.4)$ & 0.124 & 0.925 \\
\hline Maternal grandmother & $21.4(0-42.9)$ & $20.6(12.9-28.3)$ & $19.0(16.8-21.2)$ & 0.814 & 0.690 \\
\hline Any grandparent & $11.3(3.9-18.7)$ & $18.1(13.9-22.3)$ & $20.5(17.6-22.3)$ & 0.076 & 0.365 \\
\hline Any FDR or grandparent & $7.6(3.1-12.1)$ & $19.0(14.5-23.5)$ & $22.2(19.0-25.4)$ & $<0.001$ & 0.281 \\
\hline
\end{tabular}

FDR, first-degree relative.

${ }^{a} \mathrm{~A}$ comparison between children with a given relative with type 1 diabetes (type 1 diabetes + and type 2 diabetes - ) and children without a relative with diabetes (type 1 diabetes - and type 2 diabetes -).

${ }^{\mathrm{b}} \mathrm{A}$ comparison between children with a given relative with type 2 diabetes (type 1 diabetes - and type 2 diabetes + ) and children without a relative with diabetes (type 1 diabetes - and type 2 diabetes - ). 
of children 0-4.99 years, $70.9 \%$ of children 5.0-9.99 years and $64.3 \%$ of children 10.0-14.99 years, $P=0.086$ ). The distribution of HLA risk genotypes in children $<2$ years at diagnosis was similar compared with older children. The proportion of children $<2$ years and strongly increased risk was 26.7 vs $23 \%$ in older children $(P=0.514)$. Children who had an FDR with type 1 diabetes had as often a strong- or moderate-risk HLA genotype as other children (67.8 vs $68.1 \%, P=0.939)$. When analysing the effect of HLA risk genotypes on DKA a significant trend was observed showing that DKA and severe DKA were more frequent in subjects with lower risk HLA genotypes (Table 1). While comparing children with an HLA genotype conferring high or moderate risk for type 1 diabetes with those carrying a genotype defining a slightly increased, neutral or decreased risk, we observed that DKA was also more often present in the latter group (17.4 vs $23.0 \%, P=0.032$ ). In a similar analysis including children $<2$ years DKA frequency was not significantly different between the two HLA class II genotype groups $(26.8$ vs $44.4 \%$, respectively; $P=0.182$ ). The logistic regression model was applied to explain DKA including age at diagnosis as a continuous variable and several categorised variables (sex, HLA DQB1 risk groups (two categories), presence of type 1 diabetes in FDR and presence of type 2 diabetes in FDR). Significant associations were observed between DKA and absence of type 1 diabetes in FDR $(P=0.001$, OR $=3.23(95 \%$ CI $1.60-6.52))$, low-risk HLA-DQB1 genotype $(P=0.029, \mathrm{OR}=1.45(95 \% \mathrm{CI}$ 1.04-2.03)) and older age at diagnosis $(P=0.02$, $\mathrm{OR}=1.07$ (95\%CI 1.03-1.12)).

\section{Discussion}

This population-based analysis of children diagnosed with type 1 diabetes $<15$ years confirms that the presence of type 1 diabetes in an FDR protects the child from DKA at diagnosis. Interestingly, we also found that children carrying high- or moderate-risk HLA DQB1 genotypes presented less often with DKA in Finland.

Frequency of DKA was very low (7.4\%) at diagnosis of type 1 diabetes in children who had at least one FDR affected by type 1 diabetes, while in the other children, the frequency of DKA was almost three times higher $(20.5 \%)$. Similar observations were made in earlier studies $(4,6)$. A reduced frequency of DKA at diagnosis in children with affected FDRs is understandable, because these families usually know the symptoms of the disease, have equipment for self-monitoring of blood glucose and are aware of the necessity to contact a doctor without any delay to start treatment. Type 2 diabetes in FDR of children with type 1 diabetes occurs rarely, but especially type 2 diabetes among the mothers may protect against DKA in their offspring. The history of type 1 diabetes in grandparents had only a small non-significant effect on the risk for DKA in newly diagnosed children. Neither did the presence of type 2 diabetes in grandparents have any effect on the frequency of DKA in the grandchildren.

Children with HLA genotypes conferring strong or moderate risk for type 1 diabetes had lower frequency of DKA and severe DKA than subjects with other HLA class II genotypes, an observation not previously reported and the reasons remaining so far unknown. The awareness of type 1 diabetes and its symptoms might have increased recently in the Finnish population because of the ongoing prospective Type 1 Diabetes Prediction and Prevention (DIPP) study. The DIPP study was initiated in the mid-1990s in three university hospitals in Finland screening all newborn infants for HLA-conferred susceptibility to type 1 diabetes and monitoring from birth those subjects who are identified to carry HLA genotypes conferring high or moderate disease risk (17). The families, who take part in the DIPP study, have been more informed about the symptoms of type 1 diabetes. In addition, diabetes-associated autoantibodies are analysed regularly at an interval of 3-12 months. As a consequence the diagnosis of type 1 diabetes is usually made at an early stage, thus probably decreasing the risk for DKA among these children. If the DIPP study had a significant role in attenuating DKA at onset, the observed associations between HLA genotypes and DKA at diagnosis would reflect the special situation in Finland and it would be difficult to export these results to other populations. However, children who have participated in the DIPP follow-up during the prediabetic period comprise only a small minority $(<$ $5 \%$ ) of all newly diagnosed cases $<15$ years in Finland during the time-period analysed, and therefore it is unlikely that the DIPP study alone would explain the association between HLA class II genotypes and DKA.

The prevalence of type 1 diabetes among siblings $(4.9 \%)$ was higher in this study than in the earlier Childhood Diabetes in Finland (DiMe) study $(2.6 \%$; $P=0.014$ ) (18), likely reflecting the steep increase in the incidence of type 1 diabetes among Finnish children. Earlier data on the frequencies of type 1 and type 2 diabetes among grandparents is scarce. In this study, the proportion of children having a grandparent with type 1 diabetes was $6.6 \%$, a figure not previously reported from Finland.

Children $<2$ years of age at diagnosis had more often an affected FDR, particularly a father with type 1 diabetes, than older children. However, these children did not more often carry high-risk HLA genotypes. This result differs from earlier Finnish studies, in which children diagnosed before the age of 2 years carried the HLA DQB1*0201/*0302 more frequently than older children (11). The possible explanation could be the temporal change observed in the distribution of HLA genotypes in newly diagnosed children showing that the frequency of high-risk HLA genotypes has decreased among recent cohorts of children diagnosed with type 1 
diabetes (19). In children $<2$ years DKA was neither associated with class II HLA genotypes nor the presence of an affected FDR. These effects could have been significant with greater sample size, however.

In conclusion, the risk of DKA in children at the diagnosis of type 1 diabetes was independently increased by the absence of an affected family member, the presence of a low-risk HLA class II genotype and older age of the index case.

\section{Supplementary data}

This is linked to the online version of the paper at http://dx.doi.org/10. 1530/EJE-11-0376.

\section{Declaration of interest}

The authors declare that there is no conflict of interest that could be perceived as prejudicing the impartiality of the research reported.

\section{Funding}

This work was supported by National Graduate School of Clinical Investigation, Helsinki, Finland, the Foundation for Paediatric Research, Helsinki, Finland, Alma and K A Snellman Foundation, Oulu, Finland, and Special Public Grants for Medical Research at Helsinki University Hospital and Oulu University Hospital.

\section{Acknowledgements}

We are grateful to the personnel at the Paediatric Centers around Finland and the personnel of Finnish Paediatric Diabetes Register, Helsinki, Finland, for collaboration.

\section{References}

1 Levy-Marchal C, Patterson CC \& Green A. Geographical variation of presentation at diagnosis of type I diabetes in children: the EURODIAB Study. Diabetologia 200144 (Supplement 3) B75-B80. (doi:10.1007/PL00002958)

2 Samuelsson U \& Stenhammar L. Clinical characteristics at onset of type 1 diabetes in children diagnosed between 1977 and 2001 in the South-East region of Sweden. Diabetes Research and Clinical Practice 200568 49-55. (doi:10.1016/j.diabres.2004.08.002)

3 Hekkala A, Knip M \& Veijola R. Ketoacidosis at diagnosis of type 1 diabetes in children in northern Finland: temporal changes over 20 years. Diabetes Care 200730 861-866. (doi:10.2337/dc062281)

4 Rewers A, Klingensmith G, Davis C, Petitti DB, Pihoker C, Rodriquez B, Schwartz ID, Imperatore G, Williams D, Dolan LM \& Dabelea D. Presence of diabetic ketoacidosis at diagnosis of diabetes mellitus in youth: the Search for Diabetes in Youth Study. Pediatrics 2008121 e1258-e1266. (doi:10.1542/peds.20071105)

5 Vanelli M, Chiari G, Ghizzoni L, Costi G, Giacalone T \& Chiarelli F. Effectiveness of a prevention program for diabetic ketoacidosis in children. An 8-year study in schools and private practices. Diabetes Care 199922 7-9. (doi:10.2337/diacare.22.1.7)

6 Veijola R, Reijonen H, Vähäsalo P, Sabbah E, Kulmala P, Ilonen J, Åkerblom HK \& Knip M. HLA-DQB1-defined genetic susceptibility, beta cell autoimmunity, and metabolic characteristics in familial and nonfamilial insulin-dependent diabetes mellitus. Childhood Diabetes in Finland (DiMe) Study Group. Journal of Clinical Investigation 199698 2489-2495. (doi:10.1172/JCI119067)
7 Hermann R, Turpeinen H, Laine AP, Veijola R, Knip M, Simell O, Sipilä I, Åkerblom HK \& Ilonen J. HLA DR-DQ-encoded genetic determinants of childhood-onset type 1 diabetes in Finland: an analysis of 622 nuclear families. Tissue Antigens $2003 \mathbf{6 2}$ 162-169. (doi:10.1034/j.1399-0039.2003.00071.x)

8 Karjalainen J, Salmela P, Ilonen J, Surcel HM \& Knip M. A comparison of childhood and adult type I diabetes mellitus. New England Journal of Medicine 1989320 881-886. (doi:10. 1056/NEJM198904063201401)

9 Caillat-Zucman S, Garchon HJ, Timsit J, Assan R, Boitard C, DjilaliSaiah I, Bougneres P \& Bach JF. Age-dependent HLA genetic heterogeneity of type 1 insulin-dependent diabetes mellitus. Journal of Clinical Investigation 199290 2242-2250. (doi:10. 1172/JCI116110)

10 Salardi S, Zucchini S, Cicognani A, Corbelli E, Santoni R, Ragni L, Elleri D \& Cacciari E. The severity of clinical presentation of type 1 diabetes in children does not significantly influence the pattern of residual $\beta$-cell function and long-term metabolic control. Pediatric Diabetes 2003 4 4-9. (doi:10.1034/j.1399-5448.2003.00028.x) 11 Komulainen J, Kulmala P, Savola K, Lounamaa R, Ilonen J, Reijonen H, Knip M \& Åkerblom HK. Clinical, autoimmune, and genetic characteristics of very young children with type 1 diabetes. Childhood Diabetes in Finland (DiMe) Study Group. Diabetes Care 199922 1950-1955. (doi:10.2337/diacare.22.12.1950)

12 Zheng C, Zhou Z, Yang L, Lin J, Huang G, Li X, Zhou W, Wang X \& Liu Z. Fulminant type 1 diabetes mellitus exhibits distinct clinical and autoimmune features from classical type 1 diabetes mellitus in Chinese. Diabetes/Metabolism Research and Reviews 201127 70-78. (doi:10.1002/dmrr.1148)

13 Tanaka S, Kobayashi T, Nakanishi K, Koyamna R, Okubo M, Murase M, Odawara $M$ \& Inoko $H$. Association of HLA-DQ genotype in autoantibody-negative and rapid-onset type 1 diabetes. Diabetes Care 200225 2302-2307. (doi:10.2337/ diacare.25.12.2302)

14 Harjutsalo V, Podar T \& Tuomilehto J. Cumulative incidence of type 1 diabetes in 10168 siblings of Finnish young-onset type 1 diabetic patients. Diabetes $2005 \mathbf{5 4}$ 563-569. (doi:10.2337/ diabetes.54.2.563)

15 Wolfsdorf J, Craig ME, Daneman D, Dunger D, Edge J, Lee W, Rosenbloom A, Sperling M \& Hanas R. Diabetic ketoacidosis in children and adolescents with diabetes. Pediatric Diabetes 2009 10 (Supplement 12) 118-133. (doi:10.1111/j.1399-5448.2009. 00569.x)

16 Hekkala A, Reunanen A, Koski M, Knip M \& Veijola R. Agerelated differences in the frequency of ketoacidosis at diagnosis of type 1 diabetes in children and adolescents. Diabetes Care 201033 1500-1502. (doi:10.2337/dc09-2344)

17 Kupila A, Muona P, Simell T, Arvilommi P, Savolainen H, Hämäläinen AM, Korhonen S, Kimpimäki T, Sjöroos M, Ilonen J, Knip M \& Simell O. Feasibility of genetic and immunological prediction of type I diabetes in a population-based birth cohort. Diabetologia 200144 290-297. (doi:10.1007/s001250051616) 18 Tuomilehto J, Lounamaa R, Tuomilehto-Wolf E, Reunanen A, Virtala E, Kaprio EA \& Åkerblom HK. Epidemiology of childhood diabetes mellitus in Finland - background of a nationwide study of type 1 (insulin-dependent) diabetes mellitus. The Childhood Diabetes in Finland (DiMe) Study Group. Diabetologia 199235 70-76. (doi:10.1007/BF00400854)

19 Hermann R, Knip M, Veijola R, Simell O, Laine AP, Åkerblom HK, Groop PH, Forsblom C, Pettersson-Fernholm K, Ilonen J \& FinnDiane Study Group. Temporal changes in the frequencies of HLA genotypes in patients with type 1 diabetes - indication of an increased environmental pressure? Diabetologia $2003 \mathbf{4 6}$ 420-425. (doi:10.1007/s00125-003-1045)

Received 29 April 2011

Revised version received 7 August 2011

Accepted 2 September 2011 